\title{
SOLVABILITY OF SINGULAR INTEGRAL EQUATIONS WITH ROTATIONS AND DEGENERATE KERNELS IN THE VANISHING COEFFICIENT CASE*
}

\author{
L. P. CASTRO ${ }^{*} \|$, E. M. ROJAS ${ }^{\dagger}$, S. SAITOH*, , N. M. TUAN ${ }^{\ddagger}$ and P. D. TUAN ${ }^{\S}$ \\ ${ }^{*}$ CIDMA - Center for RED in Mathematics and Applications, Department of Mathematics, \\ University of Aveiro, 3810-193 Aveiro, Portugal \\ ${ }^{\dagger}$ Department of Mathematics, Pontificia Universidad Javeriana, \\ D.C. Carrera 7, No. 43 - 82, Bogotá, Colombia \\ $\ddagger$ Department of Mathematics, University of Education, Viet Nam National University, \\ G7 build., 144 Xuan Thuy rd., Cau Giay dist., Ha Noi, Viet Nam \\ $\S$ Department of Mathematics, Pedagogical College, University of Da Nang, \\ 459 Ton DucThang str., Da Nang City, Viet Nam

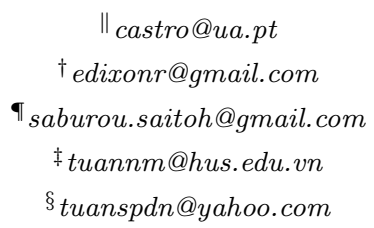

By means of Riemann boundary value problems and of certain convenient systems of linear algebraic equations, this paper deals with the solvability of a class of singular integral equations with rotations and degenerate kernel within the case of a coefficient vanishing on the unit circle. All the possibilities about the index of the coefficients in the corresponding equations are considered and described in detail, and explicit formulas for their solutions are obtained. An example of application of the method is shown at the end of the last section.

Keywords: Integral operator; singular integral equation; Riemann boundary value problem; vanishing coefficient; projection method; solvability theory.

Mathematics Subject Classification 2010: 45E10, 30E20, 30E25, 45E05, 45P05, 47A68, $47 \mathrm{G} 10$

\section{Introduction}

The theory of singular integral equations with shift (SIES) and in particular that of Cauchy singular integral operators with shift (SIOS) were studied a long time ago as

*Preprint of an article published in [Analysis and Applications 13(1) (2015), 1-21] [Article DOI: 10.1142/S0219530514500468] C [copyright World Scientific Publishing Company] [Journal URL: http://www.worldscientific.com/worldscinet/aa] 
they are applied in many fields of mathematics and physics. Particularly, the theory of SIES and boundary value problems for analytic functions founded by Hilbert and Poincaré are particularly applied in many theories such as the theory of the limit problems for differential equations with second order partial derivatives of mixed type, the theory of the cavity currents in an ideal liquid, the theory of infinitesimal bonds of surfaces with positive curvature, the contact theory of elasticity, and that of physics of plasma. Moreover, the theory of SIOS contributes theoretically in a significant way not only to the theory of Fredholm operators (Noetherian operators according to the terminology of Russian-language literature) and to that of one-sided invertible operators but also to the theory of general and abstract operators within $C^{*}$-algebras (see $[1,6,9,14,15,25,26,28,29]$ and references therein). In the previous decades, the theory of SIES has been considered an attractive object of study due to a great variety of reasons. Vekua's paper [37] was by its own means an initial motivation, and it is considered to be the first paper in which SIES were considered.

In the case where no shifts or rotations arise, the theory is already rather complete. For instance, note that the Fredholm theory of singular integral operators with piecewise continuous coefficients on composed Lyapunov curves acting on Hölder spaces (with power weights) was already constructed by R. Duduchava in the early seventies $[11,12,13]$ (cf. also the book by I. Gohberg and N. Krupnik [16]).

In recent years, many papers devoted to particular investigations and containing solutions in explicit form of SIES have been published (see $[3,4,5,8,17,18,19,20,21]$ ). The most general and important class among the SIES reducible to Riemann boundary value problems that can provide explicit solutions in a certain sense, is that of singular integral equations with a Carleman shift. However, there are only a few special types of such SIES which are possible to solve completely in some extent or by means of Riemann boundary value problems (see $[28,36]$ ). Anyway, abstract normalization procedures are known for obtaining the solutions of consequent normalized problems of singular integral operators with Carleman shifts and degenerate coefficients within the framework of Lebesgue spaces on the unit circle (or the real line); cf. [10,22,23,24,33].

In this paper, we study the solvability of a class of singular integral equations with rotations on the unit circle and with degenerate kernel in the case of a vanishing coefficient (and where the rotation is considered as a Carleman shift). This leads to an advance of the knowledge since we obtain consequent solutions for the problems in analysis which previously where only known for cases where no degenerate coefficients were occurring. Anyway, all this happens subjected to the known condition that a certain difference between the square of the coefficients (after an action of the rotations) do not vanish - which is in fact a known necessary condition for the existence of solutions. Within our method we will face a consequent linear boundary value problem for analytic functions - to which type the pioneering works of Riemann, Hilbert, Haseman, Carleman, Muskhelishvili, Gakhov and Vekua gave significant main initial contributions. 
In view to formulate our initial problem we will now fix some notation. Let $\Gamma=\{t \in \mathbb{C}:|t|=1\}$ and $X=H^{\mu}(\Gamma)$, with $0<\mu<1$, be the Hölder space on $\Gamma$. Additionally, we will consider the following operators in $X$ :

$$
\begin{aligned}
& (W \varphi)(t)=\varphi\left(\varepsilon_{1} t\right), \quad(S \varphi)(t)=\frac{1}{\pi i} \mathrm{p} \cdot \mathrm{v} \cdot \int_{\Gamma} \frac{\varphi(\tau)}{\tau-t} d \tau, \\
& (M \varphi)(t)=\frac{1}{\pi i} \int_{\Gamma} m(\tau, t) \varphi(\tau) d \tau,
\end{aligned}
$$

where $\varepsilon_{1}=\exp \left(\frac{2 \pi i}{n}\right), n \in \mathbb{N}, \varepsilon_{k}=\varepsilon_{1}{ }^{k}$ and $m(\tau, t)$ is a given function satisfying the Hölder condition in $(\tau, t) \in \Gamma \times \Gamma$. Since the operator $W$ is an involution of degree $n$, one can construct the projections $P_{j}(j=1, \ldots, n)$ satisfying the following identities:

$$
\left\{\begin{array}{l}
P_{j}=\frac{1}{n} \sum_{k=1}^{n} \varepsilon_{j}^{n-1-k} W^{k+1}, \quad j=1,2, \ldots, n, \\
P_{i} P_{j}=\delta_{i j} P_{j}, \quad i, j=1, \ldots, n \\
W^{k}=\sum_{j=1}^{n} \varepsilon_{j}^{k} P_{j}, \quad k=1, \ldots, n \\
P_{1}+P_{2}+\cdots+P_{n-1}+P_{n}=I
\end{array}\right.
$$

where $\delta_{i j}$ is the Kronecker symbol (see $[8,34]$ ).

We are now in a position to consider the main object of the present work in the form of the following singular integral equation (in $X$ ), which cannot be reduced to a two-term boundary value problem (see [28]),

$$
a(t) \varphi(t)+b(t)\left[(S+M) P_{\ell} \varphi\right](t)+\sum_{j=1}^{m} \frac{1}{\pi i} \int_{\Gamma} a_{j}(t) b_{j}(\tau) \varphi(\tau) d \tau=f(t),
$$

where $a, b, f, a_{j}, b_{j} \in X(j=1,2, \ldots, m)$ are given and $S, M, P_{\ell}(1 \leq \ell \leq n)$ are the operators defined in (1.1)-(1.2). In the general case of Carleman shift $W$, the equation of the form (1.3) attracted the attention of many authors. Namely, under the assumption that the coefficient $a(t)$ does not vanish on $\Gamma$, the papers $[3,4,7,8$, 28,31 ] studied the solvability and obtained explicit solutions of their corresponding equations (1.3) by means of Riemann boundary value problems.

Our main goal is to analyse the solvability and obtain eventual solutions of (1.3) when the function-coefficient $a(t)$ vanishes on $\Gamma$ in the sense that it has isolated zero-points, i.e.

$$
a(t)=\prod_{j=1}^{s}\left(t-\alpha_{j}\right)^{r_{j}} u(t)
$$

where $\alpha_{j} \in \Gamma, r_{j}(j=1,2, \ldots, s)$ are positive integers and $u(t)$ is a non-vanishing function on $\Gamma$. Without loss of generality we may assume from now on that $u(t) \equiv 1$. 


\section{Some lemmas and preparing results}

In order to state the main theorems, we need some preliminary and technical results. For every $a \in X$ we write $\left(K_{a} \varphi\right)(t)=a(t) \varphi(t)$.

Lemma 2.1. ([34], pp. 203) Suppose that $a \in X$ is fixed. Then for every pair $(k, j), k, j \in\{1,2, \ldots, n\}$ there exists an element $a_{k j} \in X$ such that $P_{k} K_{a} P_{j}=$ $K_{a_{k j}} P_{j} ;$ namely,

$$
a_{k j}(t)=\frac{1}{n} \sum_{\nu=1}^{n} \varepsilon_{\nu+1}^{j-k} a\left(\varepsilon_{\nu+1} t\right)
$$

Lemma 2.2. ([34]) Let $a \in X$ be fixed. Then for every pair $(k, j), k, j \in$ $\{1,2, \ldots, n\},(1.2)$ yields

$$
P_{k} K_{a_{k j}}=K_{a_{k j}} P_{j}
$$

where $a_{k j}(t)$ is defined by $(2.1)$.

We set

$$
\begin{aligned}
\Omega_{0} & =\left\{\alpha_{i}, i=1,2, \ldots, s\right\}, \\
\Omega & =\left\{t_{i \mu}=\varepsilon_{\mu+1}^{-1} \alpha_{i}, \mu=1,2, \ldots, n ; i=1,2, \ldots, s\right\} .
\end{aligned}
$$

In the sequel, let $\frac{d}{d t}$ denote the arc length derivative operation on $\Gamma$. Additionally, let us denote by $C(\Gamma)$ the set of all continuous functions on $\Gamma$, and by $C^{k}(\Gamma)$ the set of all functions which together with their derivatives (with respect to the arc length) up to the $k$-order $(k \in \mathbb{N})$ belong to $C(\Gamma)$. For every $g \in C^{k}(\Gamma)$, we use the following notation

$$
\{g(t)\}_{\left(k, t_{0}\right)}:=\left.\frac{d^{k}}{d t^{k}} g(t)\right|_{t=t_{0}} .
$$

For $g \in C^{k}(\Gamma)$ given, we write:

$$
\begin{aligned}
& g^{*}(t)=g(t) \prod_{\substack{\mu=1 \\
\mu \neq n-1}}^{n} a\left(\varepsilon_{\mu+1} t\right), \\
& g_{k}^{*}(t)=P_{k} g^{*}(t),
\end{aligned}
$$

where $a(t)$ is given by (1.4) with $u(t) \equiv 1$, and $P_{k}$ is the projection as in (1.2). Obviously, $a \in C^{q}(\Gamma)$ for $q=0,1, \ldots, r_{i}-1$.

Lemma 2.3. Suppose $g \in C^{q}(\Gamma) ; q=0,1, \ldots, r_{i}-1$. Within this assumption, $\{g(t)\}_{\left(q, \alpha_{i}\right)}=0$ for all $\alpha_{i} \in \Omega_{0}$ if and only if

$$
\left\{g_{k}^{*}(t)\right\}_{\left(q, t_{i \mu}\right)}=0 \text { for all } k=1,2, \ldots, n \text { and } t_{i \mu} \in \Omega .
$$


Proof. Suppose that $\{g(t)\}_{\left(q, \alpha_{i}\right)}=0$, for all $\alpha_{i} \in \Omega_{0}, q=0,1, \ldots, r_{i}-1$. This implies $\left\{g^{*}(t)\right\}_{\left(q, t_{i \mu}\right)}=0$, for all $t_{i \mu} \in \Omega, q=0,1, \ldots, r_{i}-1$. Hence, $\left\{g_{k}^{*}(t)\right\}_{\left(q, t_{i \mu}\right)}=$ 0 , for all $t_{i \mu} \in \Omega, q=0,1, \ldots, r_{i}-1 ; k=1,2, \ldots, n$.

Conversely, suppose that $\left\{g_{k}^{*}(t)\right\}_{\left(q, t_{i \mu}\right)}=0$, for all $t_{i \mu} \in \Omega, q=0,1, \ldots, r_{i}-$ $1 ; k=1,2, \ldots, n$. It follows that

$$
\left\{\sum_{k=1}^{n} g_{k}^{*}(t)\right\}_{\left(q, t_{i \mu}\right)}=0, \text { for all } t_{i \mu} \in \Omega, q=0,1, \ldots, r_{i}-1 .
$$

On the other hand,

$$
\begin{aligned}
\sum_{k=1}^{n} g_{k}^{*}(t) & =\sum_{k=1}^{n} \frac{1}{n} \sum_{j=1}^{n} \varepsilon_{k}^{n-1-j} g\left(\varepsilon_{j+1} t\right) \prod_{\substack{\mu=1 \\
\mu \neq j}}^{n} a\left(\varepsilon_{\mu+1} t\right) \\
& =\sum_{j=1}^{n}\left[\frac{1}{n} \sum_{k=1}^{n} \varepsilon_{k}^{n-1-j}\right] g\left(\varepsilon_{j+1} t\right) \prod_{\substack{\mu=1 \\
\mu \neq j}}^{n} a\left(\varepsilon_{\mu+1} t\right) \\
& =g(t) \prod_{\substack{\mu=1 \\
\mu \neq n-1}}^{n} a\left(\varepsilon_{\mu+1} t\right)=g^{*}(t) .
\end{aligned}
$$

We then have

$$
\left\{g^{*}(t)\right\}_{\left(q, t_{i \mu}\right)}=0, \text { for all } t_{i \mu} \in \Omega, q=0,1, \ldots, r_{i}-1 .
$$

Hence,

$$
\{g(t)\}_{\left(q, \alpha_{i}\right)}=0, \text { for all } \alpha_{i} \in \Omega_{0}, q=0,1, \ldots, r_{i}-1,
$$

and the proof is complete.

Lemma 2.4. Let $\alpha \in \Gamma$ be fixed. Assume that $h \in X$ is a function having a continuous analytic extension in a neighborhood of $\Gamma$. Then the equation

$$
(t-\alpha)^{r} \varphi(t)=h(t),
$$

has a solution in $X$ if and only if the following conditions are satisfied

$$
\{h(t)\}_{(q, \alpha)}=0, \text { for all } q=0,1, \ldots, r-1 .
$$

If this is the case, then the solution of equation (2.2) is given by

$$
\varphi(t)=\left\{\begin{array}{l}
\frac{h(t)}{(t-\alpha)^{r}} \text { if } t \neq \alpha \\
\frac{\{h(t)\}_{(r, \alpha)}}{r !} \text { if } t=\alpha .
\end{array}\right.
$$

Moreover, the solution also has an analytic extension in a neighborhood of $\Gamma$. 
Proof. Suppose that equation (2.2) has a solution $\varphi_{0} \in X$, i.e.

$$
(t-\alpha)^{r} \varphi_{0}(t)=h(t)
$$

for all $t \in \Gamma$. Expanding the function $h$ in a neighborhood of $\alpha \in \Gamma$ in a Taylor series, we have

$$
h(z)=h(\alpha)+\sum_{k=1}^{r-1} \frac{(z-\alpha)^{k}}{k !}\{h(z)\}_{(k, \alpha)}+\frac{(z-\alpha)^{r}}{r !}\{h(z)\}_{(r, \xi)},
$$

where $z$ and $\xi$ are in a neighborhood of $\Gamma$. Note that the notations $\{h(z)\}_{(k, \alpha)}$ and $\{h(t)\}_{(k, \alpha)}$ have the same meaning as $h$ is an analytic function in a neighborhood of $\Gamma$. For $t \in \Gamma$,

$$
(t-\alpha)^{r} \varphi_{0}(t)=h(\alpha)+\sum_{k=1}^{r-1} \frac{(t-\alpha)^{k}}{k !}\{h(t)\}_{(k, \alpha)}+\frac{(t-\alpha)^{r}}{r !}\{h(t)\}_{(r, \xi)}
$$

where $\xi \in \Gamma$. Deducing inductively we obtain $\{h(t)\}_{(q, \alpha)}=0$ for $q=0,1, \ldots r-1$. Thus, the condition (2.3) is necessary.

Conversely, suppose that the condition (2.3) is satisfied. We have to prove that the function $\varphi$ defined by (2.4) fulfills the Hölder condition. By the assumption we have

$$
h(z)=h(\alpha)+\sum_{k=1}^{r-1} \frac{(z-\alpha)^{k}}{k !}\{h(z)\}_{(k, \alpha)}+\frac{(z-\alpha)^{r}}{r !}\{h(z)\}_{(r, \xi)},
$$

where $z, \xi$ are in a neighborhood of $\Gamma$. From the condition (2.3), we derive

$$
h(z)=\frac{(z-\alpha)^{r}}{r !}\{h(z)\}_{(r, \xi)} .
$$

We deduce that the function $\varphi$ defined by (2.4) is Hölder continuous on the curve $\Gamma$. Therefore, $\varphi \in X$.

Thanks to the analytic extension of $h$ we assert that the function $\varphi$ as in (2.4) also has an analytic extension in a neighborhood of $\Gamma$. The proof is complete.

Write $r:=\max _{1 \leq i \leq s}\left\{r_{i}\right\}$. Note that $\alpha_{i} \in \Gamma$ are fixed points for $i=1,2, \ldots, s$. The following lemma is a direct consequence of Lemma 2.4.

Lemma 2.5. Assume that the function $h \in X$ has a continuous analytic extension in a neighborhood of $\Gamma$. The equation

$$
\prod_{i=1}^{s}\left(t-\alpha_{i}\right)^{r_{i}} \varphi(t)=h(t)
$$

has a solution if and only if the following conditions are fulfilled

$$
\{h(t)\}_{\left(q, \alpha_{i}\right)}=0, \text { for all } i=1,2, \ldots s ; q=0,1, \ldots, r_{i}-1 .
$$


If this is the case, then equation (2.8) has a unique solution given by

$$
\varphi(t)=\left\{\begin{array}{l}
\frac{h(t)}{\prod_{i=1}^{s}\left(t-\alpha_{i}\right)^{r_{i}}} \text { if } t \neq \alpha_{j}, \alpha_{j} \in \Omega_{0}, \\
\frac{\{h(t)\}_{\left(r_{j}, \alpha_{j}\right)}}{r_{j} ! \prod_{\substack{i=1 \\
i \neq j}}^{n}\left(\alpha_{j}-\alpha_{i}\right)^{r_{i}}} \text { if } t=\alpha_{j}, \alpha_{j} \in \Omega_{0} .
\end{array}\right.
$$

Moreover, the solution also has an analytic extension in a neighborhood of $\Gamma$.

\section{Reducing equation (1.3) to a system of singular integral equations}

Denote by $N_{b_{j}}(j=1, \ldots, m)$ the linear functionals on $X$ that are defined as follows

$$
\left(N_{b_{j}} \varphi\right)=\frac{1}{\pi i} \int_{\Gamma} b_{j}(\tau) \varphi(\tau) d \tau, \text { for any } \varphi \in X .
$$

Put $\left(N_{b_{j}} \varphi\right)=\lambda_{j}, j=1,2, \ldots, m$. We reduce equation (1.3) to the following problem: find solutions $\varphi$ of equation

$$
a(t) \varphi(t)+b(t)\left[(S+M) P_{\ell} \varphi\right](t)=f(t)-\sum_{j=1}^{m} \lambda_{j} a_{j}(t),
$$

depending on the parameters $\lambda_{1}, \ldots, \lambda_{m}$ subjected to

$$
\left(N_{b_{j}} \varphi\right)=\lambda_{j}, j=1,2, \ldots, m .
$$

Lemma 3.1. (see [34]) Suppose that the function $m(\tau, t)$ satisfies the condition $m(\tau, t)=m\left(\varepsilon_{1} \tau, t\right)=\varepsilon_{1}^{-1} m\left(\tau, \varepsilon_{1} t\right)$. Then $\varphi \in X$ is a solution of (3.1) if and only if $\left\{\varphi_{k}=P_{k} \varphi, k=1, \ldots, n\right\}$ is a solution of the following system

$$
a^{*}(t) \varphi_{k}(t)+b_{k \ell}^{*}(t)\left[(S+M) \varphi_{\ell}\right](t)=f_{k}^{*}(t), k=1,2, \ldots, n,
$$

where

$$
\begin{aligned}
a^{*}(t) & =\prod_{j=1}^{n} a\left(\varepsilon_{j+1} t\right), \\
b_{k \ell}^{*}(t) & =\frac{1}{n} \sum_{j=1}^{n} \varepsilon_{j+1}^{\ell-k} b\left(\varepsilon_{j+1} t\right) \prod_{\substack{\mu=1 \\
\mu \neq j}}^{n} a\left(\varepsilon_{\mu+1} t\right), \\
f_{k}^{*}(t) & =\frac{1}{n} \sum_{j=1}^{n} \varepsilon_{k}^{n-1-j}\left[f\left(\varepsilon_{j+1} t\right)-\sum_{\nu=1}^{m} \lambda_{\nu} a_{\nu}\left(\varepsilon_{j+1} t\right)\right] \prod_{\substack{\mu=1 \\
\mu \neq j}}^{n} a\left(\varepsilon_{\mu+1} t\right) .
\end{aligned}
$$


Lemma 3.2. (cf. [34]) If $\left(\varphi_{1}, \varphi_{2}, \ldots, \varphi_{n}\right)$ is a solution of the system (3.3), then $\left(P_{1} \varphi_{1}, P_{2} \varphi_{2}, \ldots, P_{n} \varphi_{n}\right)$ is also a solution of it.

Theorem 3.1. (see [34]) Suppose that the function $m(\tau, t)$ satisfies the assumption of Lemma 3.1.

(1) If $\left(\varphi_{1}, \varphi_{2}, \ldots, \varphi_{n}\right)$ is a solution of $(3.3)$, then

$$
\varphi=\sum_{k=1}^{n} P_{k} \varphi_{k}
$$

is a solution of equation (3.1).

(2) If $\varphi \in X$ is a solution of (3.1) then $\left(P_{1} \varphi, P_{2} \varphi, \ldots, P_{n} \varphi\right)$ is a solution of (3.3).

Theorem 3.2. Suppose that the function $m(\tau, t)$ satisfies the assumption of Lemma 3.1. Assume that each one of the functions $f$ and $b$ has an analytic extension in a neighborhood of $\Gamma$. Then equation (3.1) has a solution in $X$ if only if the $\ell$-th equation of (3.3),

$$
a^{*}(t) \varphi_{\ell}(t)+b_{\ell \ell}^{*}(t)\left[(S+M) \varphi_{\ell}\right](t)=f_{\ell}^{*}(t),
$$

has a solution $\varphi_{\ell}(t) \in X_{\ell}=P_{\ell} X$ satisfying the conditions

$$
\left\{f(t)-\sum_{\nu=1}^{m} \lambda_{\nu} a_{\nu}(t)-b(t)\left[(S+M) \varphi_{\ell}\right](t)\right\}_{\left(q, \alpha_{i}\right)}=0
$$

where $\alpha_{i} \in \Omega_{0}, i=1,2, \ldots, s ; q=0,1, \ldots, r_{i}-1$. Moreover, if $\varphi_{\ell}$ is a solution of equation (3.5) satisfying the conditions (3.6), then equation (3.1) has a solution given by

$$
\varphi(t)=\left\{\begin{array}{l}
\frac{f(t)-\sum_{\nu=1}^{m} \lambda_{\nu} a_{\nu}(t)-b(t)\left[(S+M) P_{\ell} \varphi_{\ell}\right](t)}{a(t)} \text { if } t \neq \alpha_{i}, \alpha_{i} \in \Omega_{0}, \\
\frac{\left\{f(t)-\sum_{\nu=1}^{m} \lambda_{\nu} a_{\nu}(t)-b(t)\left[(S+M) P_{\ell} \varphi_{\ell}\right](t)\right\}_{\left(r_{j}, \alpha_{j}\right)}}{r_{j} ! \prod_{\substack{i=1 \\
i \neq j}}^{s}\left(\alpha_{j}-\alpha_{i}\right)^{r_{i}}} \text { if } t=\alpha_{j}, \alpha_{j} \in \Omega_{0} .
\end{array}\right.
$$

Proof. It follows from Lemma 3.1 that if $\varphi \in X$ is a solution of $(3.1)$, then $\left(P_{1} \varphi, P_{2} \varphi, \ldots, P_{n} \varphi\right)$ is a solution of system (3.3). It means that $\varphi_{\ell}=P_{\ell} \varphi \in X_{\ell}$ is a solution of the $\ell$-th equation of (3.3) which is equation (3.5). Moreover, for any $k=1,2, \ldots, n, \varphi_{k}$ is a solution of the equation

$$
a^{*}(t) \varphi_{k}(t)=f_{k}^{*}(t)-b_{k \ell}^{*}(t)\left[(S+M) \varphi_{\ell}\right](t) .
$$

The function in the left-hand side of (3.8) has zeros of order $r_{i}$ at $t_{i \mu}=\varepsilon_{\mu+1}^{-1} \alpha_{i} \in \Omega$. By using the assumptions and Lemmas 2.3, 2.4, and 2.5 we derive that condition (3.6) is necessary. 
Conversely, if $\varphi_{\ell}(t) \in X_{\ell}$ is a solution of (3.5) satisfying (3.6), then by Lemma 2.3,

$$
\left\{f_{k}^{*}(t)-b_{k \ell}^{*}(t)\left[(S+M) \varphi_{\ell}\right](t)\right\}_{\left(q, t_{i \mu}\right)}=0 \text {, for all } k=1,2, \ldots, n,
$$

where $t_{i \mu} \in \Omega, i=1,2, \ldots, s ; q=0,1, \ldots, r_{i}-1$. By Lemma 2.5 , the equation

$$
a^{*}(t) \varphi_{k}(t)=f_{k}^{*}(t)-b_{k \ell}^{*}(t)\left[(S+M) \varphi_{\ell}\right](t) \text {, for some } k=1,2, \ldots, n,
$$

has a solution $\varphi_{k} \in X$.

Due to Theorem 3.1, $\varphi(t)=\sum_{k=1}^{n} P_{k} \varphi_{k}(t)$ is a solution of (3.1). Moreover, it is easily seen that $P_{\ell} \varphi=P_{\ell} \varphi_{\ell}=\varphi_{\ell}$. From equation (3.1), we obtain

$$
a(t) \varphi(t)=f(t)-\sum_{j=1}^{m} \lambda_{j} a_{j}(t)-b(t)\left[(S+M) \varphi_{\ell}\right](t) .
$$

By Lemma 2.5 we derive that (3.1) has a solution $\varphi$ determined by (3.7). The proof is complete.

Remark 3.1. Suppose that $f, g \in X$. It is clear that if $f(t)=g(t)$ for all $t \in$ $\Gamma \backslash\left\{\alpha_{1}, \alpha_{2}, \ldots, \alpha_{s}\right\}$ then $f(t)=g(t)$ for all $t \in \Gamma$. Thanks to this fact, instead of (3.7), in the sequel we simply write

$$
\varphi(t)=\frac{f(t)-\sum_{\nu=1}^{m} \lambda_{\nu} a_{\nu}(t)-b(t)\left[(S+M) P_{\ell} \varphi_{\ell}\right](t)}{a(t)} .
$$

Lemma 3.3. The equation (3.5) has a solution in $X_{\ell}$ if and only if it has a solution in $X$. Moreover, if $\varphi \in X$ is a solution of (3.5), then $\varphi_{\ell}=P_{\ell} \varphi$ is a solution of (3.5) in $X_{\ell}$.

Proof. By Lemma 2.2, $b_{\ell \ell}^{*}$ is invariant with respect to $P_{\ell}$ and the operator $S+M$ commutes with $P_{\ell}$. Suppose that $\varphi \in X$ is a solution of (3.5). Applying the operator $P_{\ell}$ to both sides of (3.5), we obtain $\varphi_{\ell}=P_{\ell} \varphi$ which is a solution of (3.5).

\section{The solvability of equation $(3.5)$}

We set

$$
D^{+}=\{z \in \mathbb{C}:|z|<1\}, \quad D^{-}=\{z \in \mathbb{C}:|z|>1\} .
$$

Denote by $H\left(D^{+}\right)$and $H\left(D^{-}\right)$the sets of analytic functions in $D^{+}$and $D^{-}$, respectively. Consider the equation (3.5)

$$
a^{*}(t) \varphi_{\ell}(t)+b_{\ell \ell}^{*}(t)\left[(S+M) \varphi_{\ell}\right](t)=f_{\ell}^{*}(t) .
$$

Suppose that the function

$$
\mathcal{M}(\tau, t)=\frac{b_{\ell \ell}^{*}(t)}{a^{*}(t)+b_{\ell \ell}^{*}(t)} m(\tau, t)
$$


admits an analytic extension onto $D^{+}$in each one of variables $\tau$ and $t$. Assume that $m(\tau, t)=m\left(\varepsilon_{1} \tau, t\right)=\varepsilon_{1}^{-1} m\left(\tau, \varepsilon_{1} t\right)$. Put

$$
\Phi_{\ell}(z)=\frac{1}{2 \pi i} \int_{\Gamma} \frac{\varphi_{\ell}(\tau)}{\tau-z} d \tau .
$$

According to the Sokhotsky-Plemelj formula, we have

$$
\begin{aligned}
\varphi_{\ell}(t) & =\Phi_{\ell}^{+}(t)-\Phi_{\ell}^{-}(t), \\
\left(S \varphi_{\ell}\right)(t) & =\Phi_{\ell}^{+}(t)+\Phi_{\ell}^{-}(t) .
\end{aligned}
$$

So, we reduce equation (3.5) to the following boundary problem: find a sectionally analytic function $\Phi_{\ell}(z)$ on $D^{+}, D^{-}$which vanishes at infinity and satisfies:

$$
\begin{aligned}
\Phi_{\ell}^{+}(t)+\frac{b_{\ell \ell}^{*}(t)}{a^{*}(t)+b_{\ell \ell}^{*}(t)} M\left[\Phi_{\ell}^{+}(t)-\right. & \left.\Phi_{\ell}^{-}(t)\right] \\
& =\frac{a^{*}(t)-b_{\ell \ell}^{*}(t)}{a^{*}(t)+b_{\ell \ell}^{*}(t)} \Phi_{\ell}^{-}(t)+\frac{f_{\ell}^{*}(t)}{a^{*}(t)+b_{\ell \ell}^{*}(t)}
\end{aligned}
$$

Using Lemma 14.1 in [30], we have $M \Phi_{\ell}^{+}(t)=0, M \Phi_{\ell}^{-}(t) \in H\left(D^{+}\right)$. Write

$$
\left\{\begin{array}{l}
\Phi^{+}(t)=\Phi_{\ell}^{+}(t)-\frac{b_{\ell \ell}^{*}(t)}{a^{*}(t)+b_{\ell \ell}^{*}(t)} M \Phi_{\ell}^{-}(t), \\
\Phi^{-}(t)=\Phi_{\ell}^{-}(t) .
\end{array}\right.
$$

Instead of finding function $\Phi_{\ell}(z)$, we determine a sectionally analytic function $\Phi(z)$ on $D^{+}, D^{-}$that vanishes at infinity and satisfies:

$$
\Phi^{+}(t)=G(t) \Phi^{-}(t)+g(t)
$$

where

$$
G(t)=\frac{a^{*}(t)-b_{\ell \ell}^{*}(t)}{a^{*}(t)+b_{\ell \ell}^{*}(t)}, g(t)=\frac{f_{\ell}^{*}(t)}{a^{*}(t)+b_{\ell \ell}^{*}(t)} .
$$

Suppose that $a^{* 2}(t)-b_{\ell \ell}^{* 2}(t)$ does not vanish on $\Gamma$. Then we have $G, g \in X$ and $G(t) \neq 0$ for any $t \in \Gamma$. Put

$$
\begin{gathered}
\kappa=\operatorname{Ind} G(t)=\frac{1}{2 \pi i} \int_{\Gamma} d \ln G(t), \\
\Gamma(z)=\frac{1}{2 \pi i} \int_{\Gamma} \frac{\ln \left[\tau^{-\kappa} G(\tau)\right]}{\tau-z} d \tau, \\
X^{+}(z)=e^{\Gamma^{+}(z)}, \quad X^{-}(z)=z^{-\kappa} e^{\Gamma^{-}(z)} .
\end{gathered}
$$

Using the results proved in [15] and [27], we get the following cases:

(1) If $\kappa>0$, then the problem (4.5) has general solutions

$$
\Phi(z)=X(z)\left[\Psi(z)+P_{\kappa-1}(z)\right]
$$


where

$$
\Psi(z)=\frac{1}{2 \pi i} \int_{\Gamma} \frac{f_{\ell}^{*}(\tau)}{X^{+}(\tau)\left(a^{*}(\tau)+b_{\ell \ell}^{*}(\tau)\right)} \frac{d \tau}{\tau-z},
$$

and

$$
P_{\kappa-1}(z)=p_{1}+p_{2} z+\cdots+p_{\kappa} z^{\kappa-1}
$$

is a polynomial of degree $\kappa-1$ with arbitrary complex coefficients.

(2) If $\kappa \leq 0$, then the necessary condition for problem (4.5) to be solvable is

$$
\int_{\Gamma} \frac{g(\tau)}{X^{+}(\tau)} \tau^{j-1} d \tau=0, j=1,2, \ldots,-\kappa
$$

Equivalently,

$$
\int_{\Gamma} \frac{f_{\ell}^{*}(\tau) \tau^{j-1}}{X^{+}(\tau)\left(a^{*}(\tau)+b_{\ell \ell}^{*}(\tau)\right)} d \tau=0, j=1,2, \ldots,-\kappa .
$$

If condition (4.10) is satisfied, then the solution is uniquely given by

$$
\Phi(z)=\frac{X(z)}{2 \pi i} \int_{\Gamma} \frac{g(\tau)}{X^{+}(\tau)} \frac{d \tau}{\tau-z}=X(z) \Psi(z) .
$$

Now we get back to equation (3.5).

Theorem 4.1. Suppose that $a^{* 2}(t)-b_{\ell \ell}^{* 2}(t)$ does not vanish on $\Gamma$, the function $\mathcal{M}(\tau, t)$ (determined by (4.1)) admits an analytic continuation on $D^{+}$in each of the variables $\tau$ and $t$, and that $m(\tau, t)=m\left(\varepsilon_{1} \tau, t\right)=\varepsilon_{1}^{-1} m\left(\tau, \varepsilon_{1} t\right)$ is satisfied.

(1) If $\kappa>0$, then the equation (3.5) has solutions $\varphi_{\ell}(t)$ which satisfy the following formula:

$$
\begin{array}{r}
(S+M) \varphi_{\ell}(t)=X^{+}(t)\left[\Psi^{+}(t)+P_{\kappa-1}(t)\right]-\frac{a^{*}(t)}{a^{*}(t)+b_{\ell \ell}^{*}(t)} M X^{-}(t)\left[\Psi^{-}(t)+P_{\kappa-1}(t)\right] \\
+X^{-}(t)\left[\Psi^{-}(t)+P_{\kappa-1}(t)\right] .
\end{array}
$$

(2) If $\kappa \leq 0$, then the equation (3.5) is solvable if the condition (4.10) is satisfied. In this case, the equation (3.5) has a unique solution which satisfies the formula (4.11), where $P_{\kappa-1}(t) \equiv 0$.

Proof. (1) From the assumption, it follows that the problem (4.5) has a solution $\Phi(t)$ determined by (4.7). Therefore, the equation (3.5) has a solution $\varphi_{\ell}(t)$. Moreover, from the Sokhotsky-Plemelj formulas (4.2) and (4.4), we get

$$
\begin{gathered}
\left(S \varphi_{\ell}\right)(t)=\Phi^{+}(t)+\frac{b_{\ell \ell}^{*}(t)}{a^{*}(t)+b_{\ell \ell}^{*}(t)}\left(M \Phi^{-}\right)(t)+\Phi^{-}(t) \\
=X^{+}(t)\left[\Psi^{+}(t)+P_{\kappa-1}(t)\right]+\frac{b_{\ell \ell}^{*}(t)}{a^{*}(t)+b_{\ell \ell}^{*}(t)} M X^{-}(t)\left[\Psi^{-}(t)\right. \\
\left.\quad+P_{\kappa-1}(t)\right]+X^{-}(t)\left[\Psi^{-}(t)+P_{\kappa-1}(t)\right] \\
\left(M \varphi_{\ell}\right)(t)=M\left(\Phi^{+}(t)-\Phi^{-}(t)\right)=-M X^{-}(t)\left[\Psi^{-}(t)+P_{\kappa-1}(t)\right] .
\end{gathered}
$$


Hence, $(S+M) \varphi_{\ell}(t)$ is determined by (4.11).

(2) This case is proved similarly to the case (1), and therefore the corresponding details are here omitted.

We would like to observe that the conditions imposed on the function $\mathcal{M}$ are in a sense a restriction which is here needed in view to obtain the corresponding solutions upon the use of the auxiliary equations exposed above.

\section{The solvability of equation (1.3)}

Theorem 3.2 and Theorem 4.1 show that if $a^{* 2}(t)-b_{\ell \ell}^{* 2}(t)$ does not vanish in $\Gamma$, the assumptions of Theorem 4.1 and the condition (3.6) are satisfied, then the equation (3.1) is solvable in a closed form. So, a relevant question to our problem is now considered in here: will a solution of (3.1) be always a solution of (1.3)? Unfortunately, the answer to this question is negative.

In this section, we shall show that a solution of (3.1) has to satisfy condition (3.2) to be a solution of equation (1.3).

Consider the following cases:

(1) If $\kappa>0$ then from Theorem 3.2 and Theorem 4.1, we have solutions of (3.1) which are determined by (3.9) where $(S+M) \varphi_{\ell}(t)$ is given by (4.11). From (3.4) and (4.8), we get

$$
\begin{aligned}
\Psi(z)= & \frac{1}{2 \pi i} \int_{\Gamma} \frac{\frac{1}{n} \sum_{j=1}^{n} \varepsilon_{\ell}^{n-1-j} f\left(\varepsilon_{j+1} \tau\right) \prod_{\substack{\mu=1 \\
\mu \neq j}}^{n} a\left(\varepsilon_{\mu+1} \tau\right)}{X^{+}(\tau)\left(a^{*}(\tau)+b_{\ell \ell}^{*}(\tau)\right)} \frac{d \tau}{\tau-z} \\
& -\frac{1}{2 \pi i} \int_{\Gamma} \frac{\frac{1}{n} \sum_{j=1}^{n} \varepsilon_{\ell}^{n-1-j} \sum_{\nu=1}^{m} \lambda_{\nu} a_{\nu}\left(\varepsilon_{j+1} \tau\right) \prod_{\substack{\mu=1 \\
\mu \neq j}}^{n} a\left(\varepsilon_{\mu+1} \tau\right)}{X^{+}(\tau)\left(a^{*}(\tau)+b_{\ell \ell}^{*}(\tau)\right)} \frac{d \tau}{\tau-z} \\
= & \Psi_{1}(z)-\sum_{\nu=1}^{m} \lambda_{\nu} A_{\nu}(z)
\end{aligned}
$$

where

$$
\begin{aligned}
& \Psi_{1}(z)=\frac{1}{2 \pi i} \int_{\Gamma}^{\frac{1}{n} \sum_{j=1}^{n} \varepsilon_{\ell}^{n-1-j} f\left(\varepsilon_{j+1} \tau\right) \prod_{\substack{\mu=1 \\
\mu \neq j}}^{n} a\left(\varepsilon_{\mu+1} \tau\right)} \frac{d \tau}{X^{+}(\tau)\left(a^{*}(\tau)+b_{\ell \ell}^{*}(\tau)\right)} \\
& A_{\nu}(z)=\frac{1}{2 \pi i} \int_{\Gamma} \frac{\frac{1}{n} \sum_{j=1}^{n} \varepsilon_{\ell}^{n-1-j} a_{\nu}\left(\varepsilon_{j+1} \tau\right) \prod_{\substack{\mu=1 \\
\mu \neq j}}^{n} a\left(\varepsilon_{\mu+1} \tau\right)}{X^{+}(\tau)\left(a^{*}(\tau)+b_{\ell \ell}^{*}(\tau)\right)} \frac{d \tau}{\tau-z}
\end{aligned}
$$

Substituting (4.9) and (5.1) into (4.11), we obtain

$$
\begin{gathered}
(S+M) \varphi_{\ell}(t)=X^{+}(t) \Psi_{1}^{+}(t)+X^{-}(t) \Psi_{1}^{-}(t)-\frac{a^{*}(t)}{a^{*}(t)+b_{\ell \ell}^{*}(t)} M X^{-}(t) \Psi_{1}^{-}(t) \\
-\sum_{\nu=1}^{m} \lambda_{\nu}\left[X^{+}(t) A_{\nu}^{+}(t)+X^{-}(t) A_{\nu}^{-}(t)\right]+\sum_{\nu=1}^{m} \lambda_{\nu} \frac{a^{*}(t)}{a^{*}(t)+b_{\ell \ell}^{*}(t)} M X^{-}(t) A_{\nu}^{-}(t)
\end{gathered}
$$




$$
+\sum_{j=1}^{\kappa} p_{j} t^{j-1}\left[X^{+}(t)+X^{-}(t)\right]-\sum_{j=1}^{\kappa} p_{j}\left[\frac{a^{*}(t)}{a^{*}(t)+b_{\ell \ell}^{*}(t)} M X^{-}(t) t^{j-1}\right] .
$$

Thus, we can rewrite (3.9) in the form

$$
\begin{gathered}
\varphi(t)=\frac{f(t)-b(t) P_{\ell}\left[X^{+}(t) \Psi_{1}^{+}(t)+X^{-}(t) \Psi_{1}^{-}(t)\right]}{a(t)} \\
+\frac{b(t) P_{\ell}\left[\frac{a^{*}(t)}{a^{*}(t)+b_{\ell \ell}^{*}(t)} M X^{-}(t) \Psi_{1}^{-}(t)\right]}{a(t)} \\
-\sum_{\nu=1}^{m} \lambda_{\nu} \frac{a_{\nu}(t)-b(t) P_{\ell}\left[X^{+}(t) A_{\nu}^{+}(t)+X^{-}(t) A_{\nu}^{-}(t)\right]}{a(t)} \\
-\sum_{\nu=1}^{m} \lambda_{\nu} \frac{b(t) P_{\ell}\left[\frac{a^{*}(t)}{a^{*}(t)+b_{\ell \ell}^{*}(t)} M X^{-}(t) A_{\nu}^{-}(t)\right]}{a(t)} \\
-\sum_{j=1}^{\kappa} p_{j} \frac{b(t) P_{\ell}\left[t^{j-1}\left[X^{+}(t)+X^{-}(t)\right]\right]}{a(t)} \\
+\sum_{j=1}^{\kappa} p_{j} \frac{b(t) P_{\ell}\left[\frac{a^{*}(t)}{a^{*}(t)+b_{\ell \ell}^{*}(t)} M X^{-}(t) t^{j-1}\right]}{a(t)},
\end{gathered}
$$

where $X(z), \Psi_{1}(z), A_{1}(z), \ldots, A_{m}(z)$ are determined by $(4.6),(5.2)$ and $p_{1}, \ldots, p_{\kappa}$ are arbitrary.

The function $\varphi$ is a solution of the equation (1.3) if it satisfies the condition (3.2), that is

$$
\left(N_{b_{k}} \varphi\right)(t)=\lambda_{k}, \quad k=1, \ldots, m .
$$

Substituting (5.4) into the condition (3.2), we obtain

$$
\lambda_{k}=d_{k}-\sum_{\nu=1}^{m} \lambda_{\nu} e_{k \nu}-\sum_{j=1}^{\kappa} p_{j} g_{k j}, \quad k=1,2, \ldots, m
$$

where

$$
\begin{aligned}
d_{k}=N_{b_{k}} & \left(\frac{f(t)-b(t) P_{\ell}\left[X^{+}(t) \Psi_{1}^{+}(t)+X^{-}(t) \Psi_{1}^{-}(t)\right]}{a(t)}\right) \\
& +N_{b_{k}}\left(\frac{b(t) P_{\ell}\left[\frac{a^{*}(t)}{a^{*}(t)+b_{\ell \ell}^{*}(t)} M X^{-}(t) \Psi_{1}^{-}(t)\right]}{a(t)}\right), \\
e_{k \nu}=N_{b_{k}} & \left(\frac{a_{\nu}(t)-b(t) P_{\ell}\left[X^{+}(t) A_{\nu}^{+}(t)+X^{-}(t) A_{\nu}^{-}(t)\right]}{a(t)}\right) \\
& +N_{b_{k}}\left(\frac{b(t) P_{\ell}\left[\frac{a^{*}(t)}{a^{*}(t)+b_{\ell \ell}^{*}(t)} M X^{-}(t) A_{\nu}^{-}(t)\right]}{a(t)}\right),
\end{aligned}
$$




$$
\begin{aligned}
g_{k j}=N_{b_{k}} & \left(\frac{b(t) P_{\ell} t^{j-1}\left[X^{+}(t)+X^{-}(t)\right]}{a(t)}\right) \\
& -N_{b_{k}}\left(\frac{b(t) P_{\ell}\left[\frac{a^{*}(t)}{a^{*}(t)+b_{\ell \ell}^{*}(t)} M X^{-}(t) t^{j-1}\right]}{a(t)}\right) .
\end{aligned}
$$

Put

$$
\begin{gathered}
\Lambda=\left(\begin{array}{c}
\lambda_{1} \\
\lambda_{2} \\
\vdots \\
\lambda_{m}
\end{array}\right)_{m \times 1}, P=\left(\begin{array}{c}
p_{1} \\
p_{2} \\
\vdots \\
p_{\kappa}
\end{array}\right)_{\kappa \times 1}, D=\left(\begin{array}{c}
d_{1} \\
d_{2} \\
\vdots \\
d_{m}
\end{array}\right)_{m \times 1}, \\
E=\left(\begin{array}{cccc}
e_{11} & e_{12} & \ldots & e_{1 m} \\
e_{21} & e_{22} & \ldots & e_{2 m} \\
\vdots & \vdots & \ddots & \vdots \\
e_{m 1} & e_{m 2} & \ldots & e_{m m}
\end{array}\right)_{m \times m}, G=\left(\begin{array}{cccc}
g_{11} & e_{12} & \ldots & g_{1 \kappa} \\
g_{21} & g_{22} & \ldots & g_{2 \kappa} \\
\vdots & \vdots & \ddots & \vdots \\
g_{m 1} & g_{m 2} & \ldots & g_{m \kappa}
\end{array}\right)_{m \times \kappa} .
\end{gathered}
$$

Now we write (5.5) in the form of a matrix condition

$$
(I+E) \Lambda=D-G P,
$$

where $I$ is the unit matrix. On the other hand, substituting (5.3) into (3.6), we get

$$
\begin{gathered}
\left\{f(t)-b(t)\left[X^{+}(t) \Psi_{1}^{+}(t)+X^{-}(t) \Psi_{1}^{-}(t)\right]+b(t) \frac{a^{*}(t)}{a^{*}(t)+b_{\ell \ell}^{*}(t)} M X^{-}(t) \Psi_{1}^{-}(t)\right. \\
-\sum_{\nu=1}^{m} \lambda_{\nu}\left(a_{\nu}(t)-b(t)\left[X^{+}(t) A_{\nu}^{+}(t)+X^{-}(t) A_{\nu}^{-}(t)\right]\right) \\
-\sum_{\nu=1}^{m} \lambda_{\nu} b(t) \frac{a^{*}(t)}{a^{*}(t)+b_{\ell \ell}^{*}(t)} M X^{-}(t) A_{\nu}^{-}(t)-\sum_{j=1}^{\kappa} p_{j} b(t) t^{j-1}\left[X^{+}(t)+X^{-}(t)\right] \\
\left.+\sum_{j=1}^{\kappa} p_{j} b(t) \frac{a^{*}(t)}{a^{*}(t)+b_{\ell \ell}^{*}(t)} M X^{-}(t) t^{j-1}\right\}_{\left(q, \alpha_{i}\right)}=0,
\end{gathered}
$$

where $\alpha_{i} \in \Omega_{0}, q=0,1, \ldots, r_{i}-1$. Put

$$
\begin{aligned}
b_{q i}= & \left\{f(t)-b(t)\left[X^{+}(t) \Psi_{1}^{+}(t)+X^{-}(t) \Psi_{1}^{-}(t)\right]\right. \\
& \left.+b(t) \frac{a^{*}(t)}{a^{*}(t)+b_{\ell \ell}^{*}(t)} M X^{-}(t) \Psi_{1}^{-}(t)\right\}_{\left(q, \alpha_{i}\right)}, \\
u_{q i}^{\nu}= & \left\{a_{\nu}(t)-b(t)\left[X^{+}(t) A_{\nu}^{+}(t)+X^{-}(t) A_{\nu}^{-}(t)\right]\right.
\end{aligned}
$$




$$
\begin{aligned}
& \left.+b(t) \frac{a^{*}(t)}{a^{*}(t)+b_{\ell \ell}^{*}(t)} M X^{-}(t) A_{\nu}^{-}(t)\right\}_{\left(q, \alpha_{i}\right)}, \\
v_{q i}^{j}= & \left\{b(t) t^{j-1}\left[X^{+}(t)+X^{-}(t)\right]-b(t) \frac{a^{*}(t)}{a^{*}(t)+b_{\ell \ell}^{*}(t)} M X^{-}(t) t^{j-1}\right\}_{\left(q, \alpha_{i}\right)} .
\end{aligned}
$$

$$
B=\left(\begin{array}{c}
b_{11} \\
b_{21} \\
\vdots \\
b_{r_{1} 1} \\
b_{12} \\
\vdots \\
b_{r_{s} s}
\end{array}\right)_{\eta \times 1} \quad, U=\left(\begin{array}{cccc}
u_{11}^{1} & u_{11}^{2} & \ldots & u_{11}^{m} \\
u_{21}^{1} & u_{21}^{2} & \ldots & u_{21}^{m} \\
\vdots & \vdots & \ddots & \vdots \\
u_{r_{1} 1}^{1} & u_{r_{1} 1}^{2} & \ldots & u_{r_{1} 1}^{m} \\
u_{12}^{1} & u_{12}^{2} & \ldots & u_{12}^{m} \\
\vdots & \vdots & \ddots & \vdots \\
u_{r_{s} s}^{1} & u_{r_{s} s}^{2} & \ldots & u_{r_{s} s}^{m}
\end{array}\right)_{\eta \times m}
$$

$$
V=\left(\begin{array}{cccc}
v_{11}^{1} & v_{11}^{2} & \ldots & v_{11}^{\kappa} \\
v_{21}^{1} & v_{21}^{2} & \ldots & v_{21}^{\kappa} \\
\vdots & \vdots & \ddots & \vdots \\
v_{r_{1} 1}^{1} & v_{r_{1} 1}^{2} & \ldots & v_{r_{1} 1}^{\kappa} \\
v_{12}^{1} & v_{12}^{2} & \ldots & v_{12}^{\kappa} \\
\vdots & \vdots & \ddots & \vdots \\
v_{r_{s} s}^{1} & v_{r_{s} s}^{2} & \ldots & v_{r_{s} s}^{\kappa}
\end{array}\right)_{\eta \times \kappa} \quad, \text { where } \eta=\sum_{i=1}^{s} r_{i}
$$

We write (5.12) in the form of a matrix condition

$$
U \Lambda=B-V P
$$

Combining (5.11) and (5.15), we can say that the function $\varphi$ determined by (3.9) is a solution of (1.3) if and only if $\left(\lambda_{1}, \lambda_{2}, \ldots, \lambda_{m}\right)$ satisfy the following matrix condition

$$
\left(\begin{array}{c}
I+E \\
U
\end{array}\right)_{(m+\eta) \times m} \times \Lambda=\left(\begin{array}{l}
D \\
B
\end{array}\right)_{(m+\eta) \times 1}-\left(\begin{array}{l}
G \\
V
\end{array}\right)_{(m+\eta) \times \kappa} \times P
$$

(2) If $\kappa \leq 0$ then from Theorem 3.2 and Theorem 4.1 it follows that the equation (3.1) has solutions if and only if the condition (4.10) satisfied. If this is the case, 
then $P_{\kappa-1}(t) \equiv 0$. So, the solutions of (3.1) are given as follows:

$$
\begin{gathered}
\varphi(t)=\frac{f(t)-b(t) P_{\ell}\left[X^{+}(t) \Psi_{1}^{+}(t)+X^{-}(t) \Psi_{1}^{-}(t)\right]}{a(t)} \\
+\frac{b(t) P_{\ell}\left[\frac{a^{*}(t)}{a^{*}(t)+b_{\ell \ell}^{*}(t)} M X^{-}(t) \Psi_{1}^{-}(t)\right]}{a(t)} \\
-\sum_{\nu=1}^{m} \lambda_{\nu} \frac{a_{\nu}(t)-b(t) P_{\ell}\left[X^{+}(t) A_{\nu}^{+}(t)+X^{-}(t) A_{\nu}^{-}(t)\right]}{a(t)} \\
-\sum_{\nu=1}^{m} \lambda_{\nu} \frac{b(t) P_{\ell}\left[\frac{a^{*}(t)}{a^{*}(t)+b_{\ell \ell}^{*}(t)} M X^{-}(t) A_{\nu}^{-}(t)\right]}{a(t)}
\end{gathered}
$$

Therefore, the solution $\varphi$ determined by (5.17) is a solution of the equation (1.3) if $\left(\lambda_{1}, \lambda_{2}, \ldots, \lambda_{m}\right)$ satisfy the following matrix condition

$$
\left(\begin{array}{c}
I+E \\
U
\end{array}\right)_{(m+\eta) \times m} \times \Lambda=\left(\begin{array}{l}
D \\
B
\end{array}\right)_{(m+\eta) \times 1} .
$$

On the other hand, substituting (3.4) into (4.10), we get

$$
d_{j}^{\prime}+\sum_{\nu=1}^{n} \lambda_{\nu} e_{j \nu}^{\prime}=0,(j \in\{1,2, \ldots,-\kappa\}),
$$

where

$$
\begin{aligned}
d_{j}^{\prime} & =\int_{\Gamma} \frac{\frac{1}{n} \sum_{\rho=1}^{n} \varepsilon_{\ell}^{n-1-\rho} f\left(\varepsilon_{\rho+1} \tau\right) \prod_{\substack{\mu \neq 1 \\
\mu \neq \rho}}^{n} a\left(\varepsilon_{\mu+1} \tau\right)}{X^{+}(\tau)\left(a^{*}(\tau)+b_{\ell \ell}^{*}(\tau)\right)} \tau^{j-1} d \tau, \\
e_{j \nu}^{\prime} & =\int_{\Gamma} \frac{\frac{1}{n} \sum_{\rho=1}^{n} \varepsilon_{\ell}^{n-1-\rho} a_{\nu}\left(\varepsilon_{\rho+1} \tau\right) \prod_{\substack{\mu \neq 1 \\
\mu \neq \rho}}^{n} a\left(\varepsilon_{\mu+1} \tau\right)}{X^{+}(\tau)\left(a^{*}(\tau)+b_{\ell \ell}^{*}(\tau)\right)} \tau^{j-1} d \tau .
\end{aligned}
$$

Put

$$
D^{\prime}=\left(\begin{array}{c}
d_{1}^{\prime} \\
d_{2}^{\prime} \\
\vdots \\
d_{-\kappa}^{\prime}
\end{array}\right)_{-\kappa \times 1}, E^{\prime}=\left(\begin{array}{cccc}
e_{11}^{\prime} & e_{12}^{\prime} & \ldots & e_{1 m}^{\prime} \\
e_{21}^{\prime} & e_{22}^{\prime} & \ldots & e_{2 m}^{\prime} \\
\vdots & \vdots & \ddots & \vdots \\
e_{-\kappa 1}^{\prime} & e_{-\kappa 2}^{\prime} & \ldots & e_{-\kappa m}^{\prime}
\end{array}\right)_{-\kappa \times m}
$$

We write (5.19) in the form of a matrix condition

$$
E^{\prime} \Lambda=D^{\prime}
$$

Combining (5.18) and (5.21), we can say that the function $\varphi$ determined by $(5.17)$ is a solution of (1.3) if and only if $\left(\lambda_{1}, \lambda_{2}, \ldots, \lambda_{m}\right)$ satisfy the following matrix condition

$$
\left(\begin{array}{c}
I+E \\
U \\
E^{\prime}
\end{array}\right)_{(m+\eta-\kappa) \times m} \times \Lambda=\left(\begin{array}{c}
D \\
B \\
D^{\prime}
\end{array}\right)_{(m+\eta-\kappa) \times 1}
$$


Remark 5.1. Among the matrices $D, E, G, B, U, V, D^{\prime}, E^{\prime}$, the three matrices $D, B, D^{\prime}$ depend on $f(t)$; the remaining ones are completely determined by $a(t), b(t), a_{1}(t), \ldots, a_{m}(t), b_{1}(t), \ldots, b_{m}(t)$.

Theorem 5.1. Suppose that $a^{* 2}(t)-b_{\ell \ell}^{* 2}(t) \neq 0$ for any $t \in \Gamma$ and the assumptions of Theorem 4.1 are satisfied.

(1) Case $\kappa>0$. Put

$$
r=\operatorname{rank}\left(\begin{array}{cc}
I+E & G \\
U & V
\end{array}\right)_{(m+\eta) \times(m+\kappa)},
$$

where $E, G, U, V$ are determined by (5.10), (5.13) and (5.14). Then, the equation (1.3) is solvable if and only if the function $f(t)$ is such that

$$
\operatorname{rank}\left(\begin{array}{ccc}
I+E & G & D \\
U & V & B
\end{array}\right)_{(m+\eta) \times(m+\kappa+1)}=r .
$$

If this is the case, the solutions of the equation (1.3) are given by the formula (5.4), where $\left(\lambda_{1}, \ldots, \lambda_{m}\right),\left(p_{1}, \ldots, p_{\kappa}\right)$ satisfy (5.16). Moreover, we can choose $m+\kappa-r$ coefficients in $\left(\lambda_{1}, \ldots, \lambda_{m}, p_{1}, \ldots, p_{\kappa}\right)$ which are arbitrary so that $\varphi(t)$ is uniquely determined by these coefficients.

(2) Case $\kappa \leq 0$. Put

$$
r=\operatorname{rank}\left(\begin{array}{c}
I+E \\
U \\
E^{\prime}
\end{array}\right)_{(m+\eta-\kappa) \times m}
$$

where $E^{\prime}$ is determined by (5.20). Then, the equation (1.3) is solvable if and only if the function $f(t)$ determines $D, B$ and $D^{\prime}$ by the formulas (5.9), (5.13) and (5.20) which satisfy the following matrix condition

$$
\operatorname{rank}\left(\begin{array}{cc}
I+E & D \\
U & B \\
E^{\prime} & D^{\prime}
\end{array}\right)_{(m+\eta-\kappa) \times(m+1)}=r .
$$

If this is the case, the solutions of the equation (1.3) are given by the formula (5.17), where $\left(\lambda_{1}, \ldots, \lambda_{m}\right)$ satisfy (5.22). In particular, if $r=m$ and the condition (5.23) is satisfied then the equation (1.3) has a unique solution.

Proof. We will start by case (1). From the assumption it follows that the equation (1.3) has solutions if and only if there are $\left(\lambda_{1}, \ldots, \lambda_{m}\right)$ and $\left(p_{1}, \ldots, p_{\kappa}\right)$ satisfying the condition (5.16). We can rewrite (5.16) in the form

$$
\left(\begin{array}{cc}
I+E & G \\
U & V
\end{array}\right)_{(m+\eta) \times(m+\kappa)} \times\left(\begin{array}{l}
\Lambda \\
P
\end{array}\right)_{(m+\kappa) \times 1}=\left(\begin{array}{l}
D \\
B
\end{array}\right)_{(m+\eta) \times 1} .
$$


This shows that the equation (1.3) has solutions if and only if the system (5.24) has solutions in the space $\mathbb{C}^{m+\kappa}$, that is

$$
\left(\begin{array}{ccc}
I+E & G & D \\
U & V & B
\end{array}\right)_{(m+\eta) \times(m+\kappa+1)}=\left(\begin{array}{ccc}
I+E & G \\
U & V
\end{array}\right)_{(m+\eta) \times(m+\kappa)}=r .
$$

If this is the case, the system (5.24) has solutions depending on $m+\kappa-r$ coefficients. Hence, we can choose $m+\kappa-r$ coefficients in $\left(\lambda_{1}, \ldots, \lambda_{m}, p_{1}, \ldots, p_{\kappa}\right)$ so that the remaining coefficients are uniquely determined by these coefficients.

The case (2) is proved analogously to the previous case and so the details are here omitted.

\section{Example}

In this section, we consider the following particular equation in order to illustrate our method:

$$
\begin{aligned}
(t-i) \varphi(t)+\frac{-t+3 i}{2 \pi i} \int_{\Gamma} & {\left[\frac{1}{\tau-t}-\frac{1}{\tau+t}+2 t \tau^{2}\right] \varphi(\tau) d \tau } \\
& +\frac{1}{\pi i} \int_{\Gamma}[t+2+t(\tau-3)+\tau+1] \varphi(\tau) d \tau=2 t+3 .
\end{aligned}
$$

Note that the equation (6.1) is of the form of equation (1.3), where

$$
\begin{array}{r}
n=2, m=3, \ell=1, a(t)=t-i, b(t)=-t+3 i, f(t)=2 t+3, a_{1}(t)=t+2, \\
a_{2}(t)=t, a_{3}(t)=1, b_{1}(t)=1, b_{2}(t)=t-3, b_{3}(t)=t+1, m(\tau, t)=\tau^{2} t .
\end{array}
$$

Moreover, a straightforward computation allow us to identify that

$$
\begin{aligned}
& \kappa=\operatorname{Ind} G(t)=\operatorname{Ind}\left(-\frac{t^{2}+2}{2}\right)=0, \\
& \operatorname{rank}\left(\begin{array}{c}
I+E \\
U
\end{array}\right)=\operatorname{rank}\left(\begin{array}{ccc}
1 & 0 & 0 \\
0 & 1 & 0 \\
0 & 0 & 1 \\
0 & 0 & 0
\end{array}\right)=3, \\
& \operatorname{rank}\left(\begin{array}{cc}
I+E & D \\
U & B
\end{array}\right)=\operatorname{rank}\left(\begin{array}{llll}
1 & 0 & 0 & 0 \\
0 & 1 & 0 & 0 \\
0 & 0 & 1 & 0 \\
0 & 0 & 0 & 0
\end{array}\right)=3 .
\end{aligned}
$$

Having in mind Theorem 5.1, it follows that the equation (1.3) has a unique solution $\varphi(t)$ determined by (5.17), where $\lambda_{1}, \lambda_{2}, \lambda_{3}$ satisfy (5.22), that is $\lambda_{1}=\lambda_{2}=\lambda_{3}=0$. From the formula (5.17), we conclude that the equation (6.1) has a unique solution $\varphi$ given by

$$
\varphi(t)=-\frac{2 i+3}{2} t+3 i, \quad t \in \Gamma
$$




\section{Acknowledgement}

L. P. Castro and S. Saitoh were supported in part by Portuguese funds through the CIDMA - Center for Research and Development in Mathematics and Applications, and the Portuguese Foundation for Science and Technology ("FCT-Fundação para a Ciência e a Tecnologia"), within project PEst-OE/MAT/UI4106/2014.

E. M. Rojas was sponsored by the Pontificia Universidad Javeriana under Grant no. 004281.

N. M. Tuan was supported partially by the Vietnam National Foundation for Science and Technology Developments (NAFOSTED).

\section{References}

[1] M. A. Bastos, C. A. Fernandes and Y. I. Karlovich, Spectral measures in $\mathrm{C}^{*}$-algebras of singular integral operators with shifts, J. Funct. Anal. 242 (2007) 86-126.

[2] A. A. Baturev, V. G. Kravchenko and G. Litvinchuk, Approximate methods for singular integral equations with a non-Carleman shift, J. Integral Equations Appl. 8 (1996) 1-17.

[3] L. P. Castro and E. M. Rojas, On the solvability of singular integral equations with reflection on the unit circle, Integr. Equ. Oper. Theory 70 (2011) 63-99.

[4] L. P. Castro and E. M. Rojas, Explicit solutions of Cauchy singular integral equations with weighted Carleman shift, J. Math. Anal. Appl. 371 (2010) 128-133.

[5] L. P. Castro and E. M. Rojas, Invertibility of singular integral operators with flip through explicit operator relations, in Integral Methods in Science and Engineering, eds. C. Constanda and M. E. Pérez, Vol. 1 (Birkhäuser, Boston, MA, 2010), pp. 105114.

[6] L. P. Castro and E. M. Rojas, Reduction of singular integral operators with flip and their Fredholm property, Lobachevskii J. Math. 29 (2008) 119-129.

[7] L. H. Chuan and Ng. M. Tuan, On the singular integral equations with Carleman shift in the case of the vanishing coefficient, Acta Mathematica Vietnamica 28 (2003) $319-333$

[8] L. H. Chuan, N. V. Mau and N. M. Tuan, On a class of singular integral equations with the linear fractional Carleman shift and the degenerate kernel, Complex Var. Elliptic Equ. 53 (2008) 117-137.

[9] K. F. Clancey and I. Gohberg, Factorization Matrix Functions and Singular Integral Operators, Operator Theory: Advances and Applications, Vol. 3 (Birkhäuser, BaselBoston-Stuttgart, 1981).

[10] G. N. Drekova and V. G. Kravchenko, To the theory of normalization of the Riemann problem, Izv. Vyssh. Uchebn. Zaved. Mat. 9 (1991) 20-28 (in Russian).

[11] R. V. Duduchava, On boundedness of the operator of singular integration in weighted Hölder spaces, Matem. Issledov. 5(1) (1970) 56-76 (in Russian).

[12] R. V. Duduchava, Singular integral equations in weighted Hölder spaces, Matem. Issledov. 5(3) (1970) 58-82 (in Russian).

[13] R. V. Duduchava, On algebras of singular integral operators on weighted Hölder functions, Soobchenia AN Gruz. SSR 65(1) (1972) 23-26 (in Russian).

[14] J. Ferreira, G. S. Litvinchuk and M. D. L. Reis, Calculation of the defect numbers of the generalized Hilbert and Carleman boundary value problems with linear fractional Carleman shift, Integr. Equ. Oper. Theory 57 (2007) 185-207.

[15] F. D. Gakhov, Boundary Value Problems (Dover Pub., New York, 1990). 
[16] I. Gohberg and N. Krupnik, One-Dimensional Linear Singular Integral Equations, Vols. I and II (Birkhäuser Verlag, Basel, 1992).

[17] V. G. Kravchenko and G. S. Litvinchuk, Singular integral equations with Carleman linear fractional shift, Complex Variables: Theory and Applications 26 (1994) 69-78.

[18] V. G. Kravchenko and A. K. Shaev, The theory of solvability of singular integral equations with a linear-fractional Carleman shift, Soviet Math. Dokl. 43(1) (1991) $73-77$.

[19] N. Karapetiants and S. Samko, Singular integral equations on the real line with a fractional-linear Carleman shift, Proc. A. Razmadze Math. Inst. 124 (2000) 73-106.

[20] N. Karapetiants and S. Samko, Equations with Involutive Operators (Birkhäuser, Boston, 2001)

[21] A. A. Karelin, Applications of operator equalities to singular integral operators and to Riemann boundary value problems, Math. Nachr. 280 (2007) 1108-1117.

[22] V. G. Kravchenko, On the normalization of singular integral operators, Dokl. Akad. Nauk SSSR 285(6) (1985) 1314-1317 (in Russian); Soviet Math. Dokl. 32(3) (1985) 880-883 (in English).

[23] V. G. Kravchenko, A. B. Lebre and G. S. Litvinchuk, A normalization problem for a class of singular integral operators with Carleman shift and unbounded coefficients, Integr. Equ. Oper. Theory 21 (1995) 342-354.

[24] V. G. Kravchenko and G. S. Litvinchuk, On the problem of normalization of singular integral operators with shift, Uspekhi Mat. Nauk 39 (1984) 126-126 (in Russian).

[25] V. G. Kravchenko, A. B. Lebre and J. S. Rodriguez, Factorization of singular integral operators with a Carleman shift via factorization of matrix functions: the anticommutative case, Math. Nachr. 280 (2007) 1157-1175.

[26] V. G. Kravchenko and G. S. Litvinchuk, Introduction to the Theory of Singular Integral Operators with Shift (Kluwer, Amsterdam, 1994).

[27] G. S. Litvinchuk, Boundary Value Problems and Singular Integral Equations with Shift (Nauka, Moscow, 1977).

[28] G. S. Litvinchuk, Solvability Theory of Boundary Value Problems and Singular Integral Equations with shift, Mathematics and its Applications, Vol. 523 (Kluwer, Dordrecht, 2000).

[29] G. S. Litvinchuk and I. M. Spitkovsky, Factorization of Measurable Matrix Functions, Operator Theory: Advances and Applications, Vol. 70 (Birkhäuser, Basel-Boston, 2011).

[30] Ng. V. Mau, Generalized Algebraic Elements and Linear Singular Integral Equations with Transformed Arguments (WPW, Warsaw, 1989).

[31] Ng. V. Mau, On the solvability in closed form of the class of the complete singular integral equations, Diff. Equations, USSR 25 (1989) 307-311.

[32] Ng. V. Mau and Ng. M. Tuan, On solutions of integral equations with analytic kernels and rotations, Annale Polonici Mathematici LXIII (1996) 293-300.

[33] S. Prössdorf, Some Classes of Singular Equations (North-Holland Publishing Company, Amsterdam, 1978).

[34] Ng. M. Tuan, On a class of singular integral equations with rotations, Acta Mathematica Vietnamica 21 (1996) 201-211.

[35] Ng. M. Tuan, On solvability of a class of singular integral equations with rotation, Vietnam Journal of Mathematic 24 (1996) 390-397.

[36] N. L. Vasilevski and G. S. Litvinchuk, Theory of the solvability of a certain class of singular integral equations with involution, Dokl. Akad. Nauk SSSR 221 (1975) 269-271 (in Russian); Soviet Math. Dokl. 16 (1975) 318-321 (in English).

[37] N. P. Vekua, On a generalized system of singular integral equations, Soobščeniya 
Akad. Nauk Gruzin. SSR. 9 (1948) 153-160 (in Russian). 